\title{
Experience with 7.0 T MRI in Patients with Supratentorial Meningiomas
}

\author{
Sang Woo Song, M.D., ${ }^{1,2}$ Young Don Son, Ph.D., ${ }^{3}$ Zang-Hee Cho, M.D., Ph.D., ${ }^{3}$ Sun Ha Paek, M.D., Ph.D. ${ }^{1,4}$ \\ Department of Neurosurgery, ${ }^{1}$ Seoul National University College of Medicine, Seoul, Korea \\ Department of Neurosurgery, ${ }^{2}$ Konkuk University Medical Center, Seoul, Korea \\ Neuroscience Research Institute, ${ }^{3}$ Gachon University of Medicine and Science, Incheon, Korea \\ Department of Neurosurgery, ${ }^{4}$ Seoul National University Hospital, Seoul, Korea
}

\begin{abstract}
Meningiomas are typically diagnosed by their characteristic appearance on conventional magnetic resonance imaging (MRI). However, detailed image findings regarding peri- and intra-tumoral anatomical structures, tumor consistency and vascularity are very important in pre-surgical planning and surgical outcomes. At the 7.0 T MRI achieving ultra-high resolution, it could be possible to obtain more useful information in surgical strategy. Four patients who were radiologically diagnosed with intracranial meningioma in 1.5 T MRI underwent a 7.0 T MRI. Three of them underwent surgery afterwards, and one received gamma knife radiosurgery. In our study, the advantages of 7.0 T MRI over 1.5 T MRI were a more detailed depiction of the peri- and intra-tumoral vasculature and a clear delineation of tumor-brain interface. In the safety issues, all patients received $7.0 \mathrm{~T}$ MRI without any adverse event. One disadvantage of 7.0 T MRI was the reduced image quality of skull base lesions. 7.0 T MRI in patients with meningiomas could provide useful information in surgical strategy, such as the peri-tumoral vasculature and the tumor-brain interface.
\end{abstract}

Key Words : 7.0 T MRI · Meningioma.

\section{INTRODUCTION}

Meningiomas are typically diagnosed by their characteristic appearance on conventional magnetic resonance imaging (MRI). However, detailed image findings regarding peri- and intra-tumoral anatomical structures, tumor consistency and vascularity are very important in pre-surgical planning and surgical outcomes.

Since its clinical application in the 1980s, high-field MRIs have been progressively developed to improve the signal-tonoise ratio and contrast-to-noise ratio to yield higher-quality human brain images ${ }^{2,11)}$. The clinical use of higher-field MRIs is limited to $3.0 \mathrm{~T}$ because of concerns about safety ${ }^{10)}$. Recently, experimental studies using 7.0 T MRI have been performed, and there have been a few reports of MRI using high-field magnets for the visualization of brain tumors ${ }^{4,8,9)}$.

To the best of our knowledge, however, no report has explored the efficacy of 7.0 T MRI in intracranial meningioma. In this paper, we report our experiences with 7.0 T MRI in four cases of intracranial meningiomas.

\section{Procedure for 7.0 T MRI}

The 7.0 T MRI device (Magnetom 7.0T, Siemens ${ }^{\circledR}$, Munich, Germany) at the Neuroscience Research Institute of Gachon University of Medicine and Science was used for imaging.

The 7.0 T magnet, with a clear bore of $90 \mathrm{~cm}$, is equipped with a water-cooled gradient and RF coils. The gradient system operates at $2000 \mathrm{~V} / 650 \mathrm{Amps}$, with a gradient amplitude of 40 $\mathrm{mT} / \mathrm{m}$, a maximum slew rate of $200 \mathrm{mT} / \mathrm{m} / \mathrm{ms}$, and a minimum gradient rise time of 200 microseconds. A home-made 8-channel TX/RX coil was used. The scanning parameters used in each image sequence are shown in Table 1. T1-weighted MRI was performed before and after the injection of a contrast agent. Magnevist (Bayer Healthcare Pharmaceuticals Inc., Berlin, Germany) was used as the contrast agent $(0.01 \mathrm{mmol} / \mathrm{kg})$.

\section{CASE REPORT}

Four patients underwent 7.0 T MRI before treatment between April 2009 and July 2009. All of them received 1.5 T MRI at 5 to 9 days before receiving 7.0 T MRI. Three of them under-

- Received : April 9, 2015 • Revised : October 21, 2015 • Accepted : October 27, 2015

- Address for reprints : Sun Ha Paek, M.D., Ph.D.

Department of Neurosurgery, Seoul National University College of Medicine, 101 Daehak-ro, Jongno-gu, Seoul 03080, Korea

Tel : +82-2-2072-3993, Fax : +82-2-744-8459, E-mail : paeksh@snu.ac.kr

- This is an Open Access article distributed under the terms of the Creative Commons Attribution Non-Commercial License (http://creativecommons.org/licenses/by-nc/3.0) which permits unrestricted non-commercial use, distribution, and reproduction in any medium, provided the original work is properly cited. 


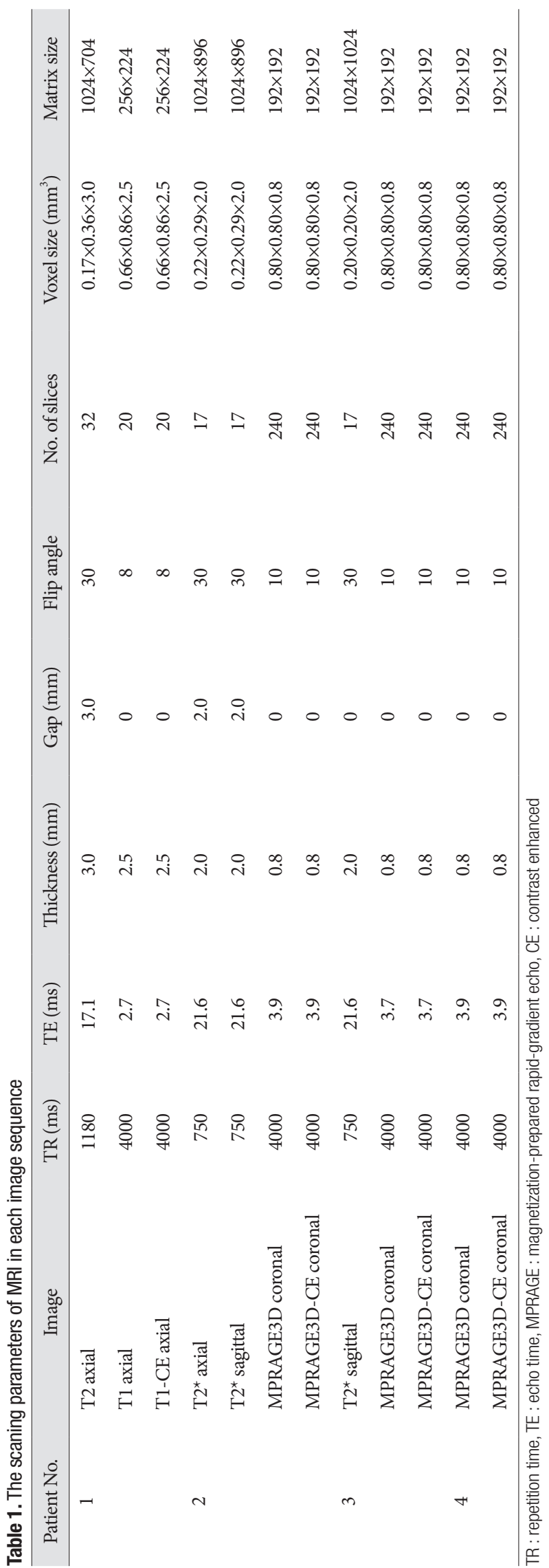

went surgery afterwards, and one received stereotactic radiosurgery. The pathological examination of surgical specimens revealed one benign fibrous meningioma and two atypical meningiomas. The patient who received radiosurgery was diagnosed with meningioma on the basis of MRI findings, such as a dural tail sign and extra-axial location.

All patients answered questionnaires after performing 7.0 T MRI. The survey asked whether the patient experienced following symptoms or not : claustrophobia; nausea; vertigo; headache; loss of balance; feeling of electric shocks or heating; skeletal muscle contractions; others. Overall, 7.0 T MRI with contrast enhancement can be safely performed in all patients without any adverse event.

\section{Case 1}

A 30-year-old woman presented with seizure and underwent brain MRI. On 1.5 T MRI, a well-enhanced mass was observed in the left parieto-occipital lobe (Fig. 1A, 2A). Surgical resection was chosen, and 29 days prior to surgery, 7.0 T MRI was also performed (Fig. 1C, 2C). Cerebral angiography showed that the middle meningeal artery was the tumor-feeding artery (Fig. 1B). Gross total resection was performed and pathological examination revealed a fibrous meningioma.

In Fig. 1C, a 7.0 T MRI shows the details of the intra- and peri-tumoral vasculature in a T2-weighted image compared with 1.5 T MRI (Fig. 1A). As shown in Fig. 2, the tumor-brain interface was more clearly delineated in 7.0 T MRI. The 7.0 T MRI showed that peri-tumoral edema had developed from areas without a cerebrospinal fluid cleft (Fig. 2C), but this finding could not be observed in $1.5 \mathrm{~T}$ MRI (Fig. 2A). Intraoperatively, some areas in which the tumor-brain interface was less obvious (Fig. 2B) were in line with the findings of 7.0 T MRI.

\section{Case 2}

A 41-year-old woman complained of a three-month history of recurrent seizure and was investigated with brain MRI. The 1.5 T MRI showed a well-enhanced mass in the right frontal lobe (Fig. 3A). Surgical resection was planned, and 23 days prior to surgery, she received 7.0 T MRI (Fig. 3C, D). Cerebral angiogram showed that enlarged middle meningeal arteries supplied the center of the tumor (Fig. 3B). Preoperative embolization was performed before subtotal resection of the tumor. The tumor was not removed from inside the superior sagittal sinus because of the risks of venous thrombosis. The pathological diagnosis was atypical meningioma.

The vessel appearance in 7.0 T MRI (Fig. 3D) closely resembled angiography, which is an accepted gold-standard mechanism of intra-cerebral vessel visualization (Fig. 3B).

\section{Case 3}

A 58-year-old man was referred to our hospital due to a mass in the right frontal convexity, which was radiologically diagnosed at the time as meningioma. Subsequently, he received ste- 
reotactic radiosurgery. At that time, the tumor size was $0.95 \mathrm{~cm}^{3}$, and he received a dose of $15 \mathrm{~Gy}$, which was prescribed to the $50 \%$ isodose line. However, the mass gradually grew to $1.5 \mathrm{~cm}^{3}$ in the next 3 years (Fig. 4A). Surgical removal was decided, and 4 days before the surgery, he received 7.0 T MRI (Fig. 4B). On pathology, the tumor was confirmed as atypical meningioma.

In this case, 7.0 T MRI showed peri-tumoral vasculature, including the cortical draining veins (Fig. 4B), in the T2-wighted image.

\section{Case 4}

A 37-year-old woman presented with a 2-year history of progressive visual loss. The $1.5 \mathrm{~T}$ brain MRI revealed a well-enhanced mass in the right orbital apex (Fig. 5A). The mass had a wide dural base at the orbital apex. It was invading the cavernous sinus and encasing the internal cerebral artery. She received 7.0 T MRI (Fig. 5B) and was subsequently recommended for surgery but voluntarily opted for radiosurgery.

In this case, the image quality of skull base lesions on $7.0 \mathrm{~T}$
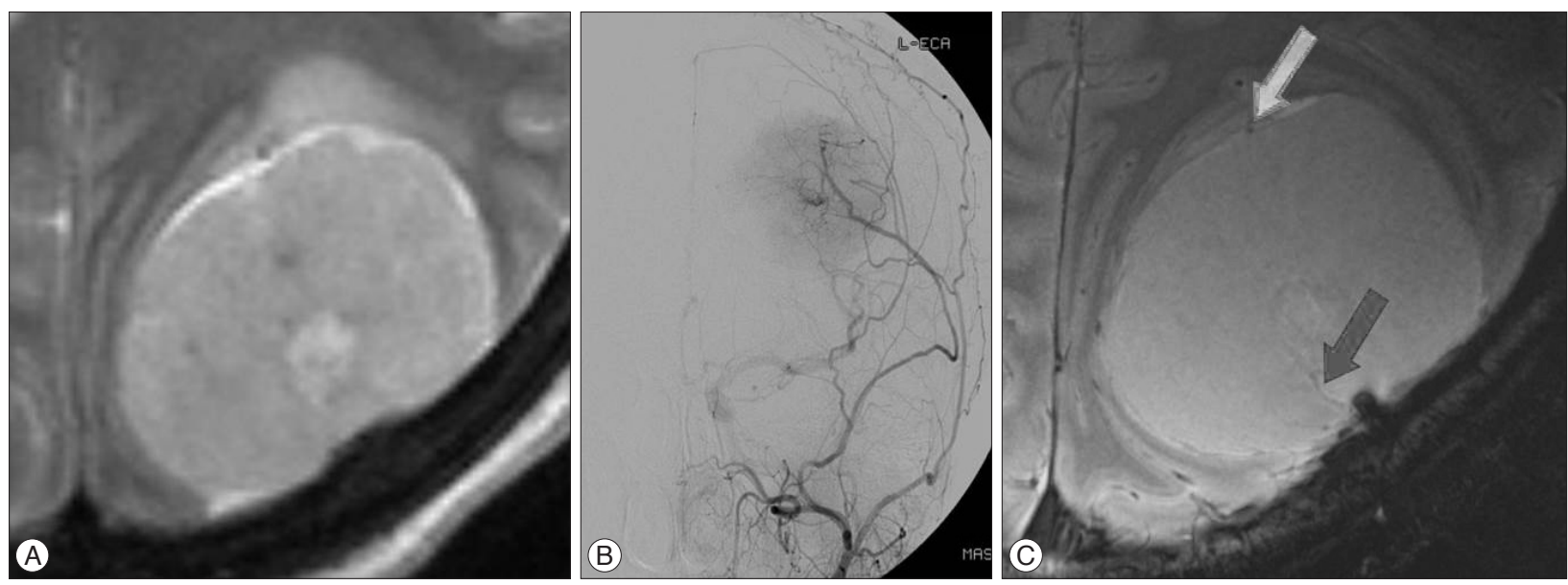

Fig. 1. A : A trans-axial T2-weighted image on 1.5 T MRI shows a left parieto-occipital, extra-axial mass. B : A left external carotid angiogram shows the arterial supply of the tumor from the middle meningeal artery. $\mathrm{C}$ : The intra-tumoral vasculature (closed arrow) and peri-tumoral vasculature (open arrow) are well delineated in 7.0 T MRI.
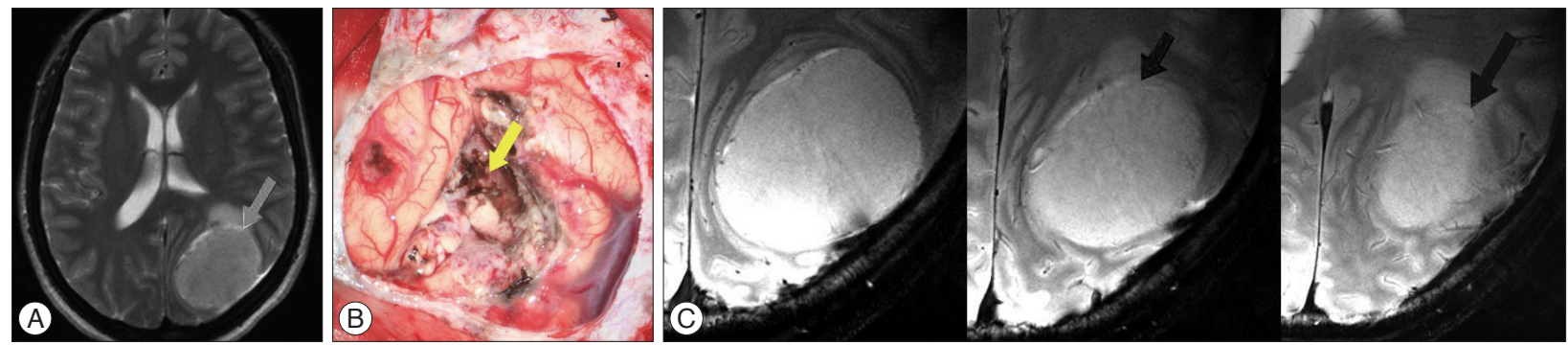

Fig. 2. A : T2-weighted axial image in case 1 shows focal peritumoral edema. In this area, a cerebrospinal fluid cleft is present, and the tumor-brain interface seems to be intact on 1.5 T MRI (closed arrow). B : Intraoperatively, some areas in which the tumor-brain interface was less obvious (open arrow). C : Serial T2-weighted images in 7.0 T MRI provide a more detailed description about the tumor-brain interface. On 7.0 T MRI, the cerebrospinal fluid cleft is not present around the peri-tumoral edema (arrow), and this result is consistent with intraoperative findings.
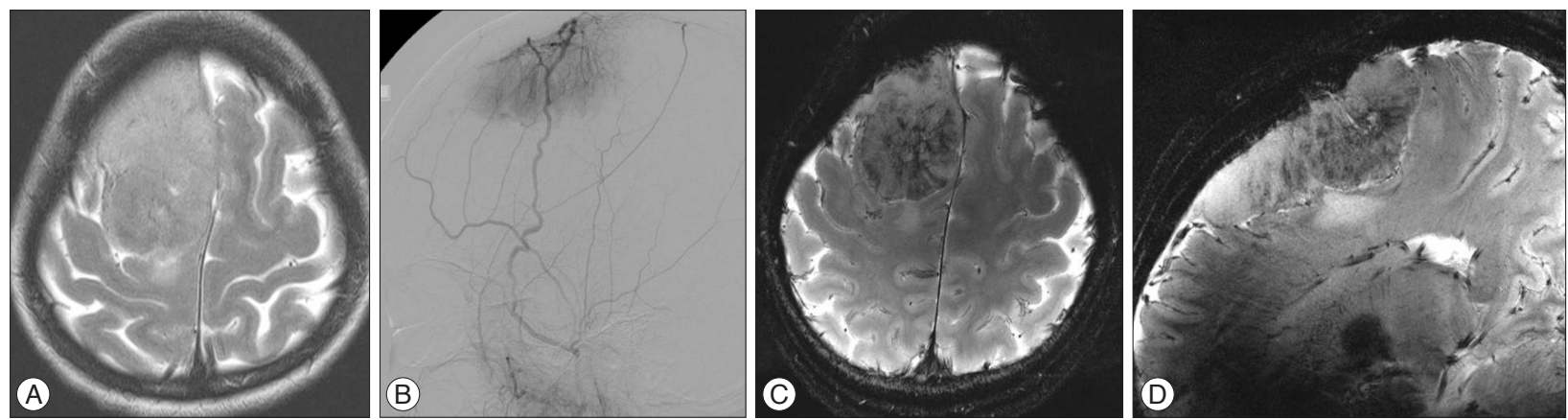

Fig. 3. A : The 1.5 T MRI shows an extra-axial mass in the right frontal lobe. $B$ : The right external carotid angiogram shows that enlarged middle meningeal arteries supply the center of a convex meningioma. C : A T2-weighted axial image on 7.0 T MRI shows more detailed intra-tumoral vasculature than 1.5 T MRI. D : The vessel appearance in T2-weighted sagittal 7.0 T MRI closely resembles angiography. 


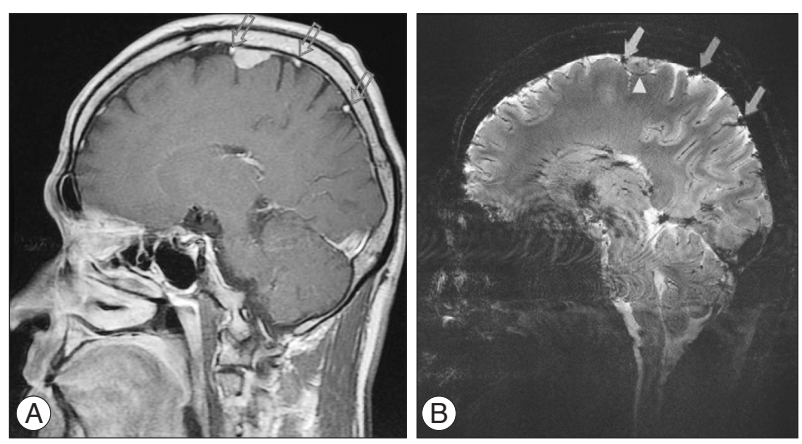

Fig. 4. A : An axial contrast-enhanced T1-weighted image on 1.5 T MRI shows a cortical draining vein (open arrows). B : A T2-weighted image on 7.0 T MRI provides detailed peri-tumoral vasculature, including cortical draining veins (closed arrows) and a vein attached to the tumor capsule (arrowhead).
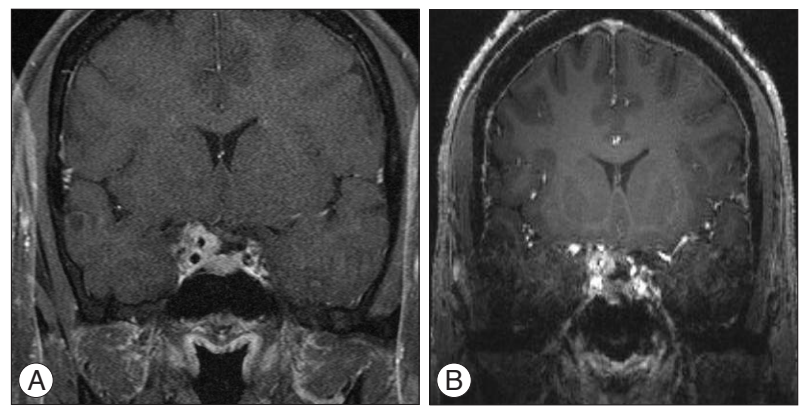

Fig. 5. A : Coronal contrast-enhanced T1-weighted image on 1.5 T MRI shows a well-enhanced mass, which invades the cavernous sinus and encases the internal carotid artery. B : An image of the anatomy below the level of the sylvian fissure is compromised by artifacts in 7.0 T MRI.

MRI was reduced compared with $1.5 \mathrm{~T}$ MRI (Fig. 5). The image of the anatomy below the level of the sylvian fissure was compromised by artifacts caused by carotid forks and the air-bone interface in 7.0 T MRI (Fig. 5B).

\section{DISCUSSION}

There have been a few reports on MRI using high-field magnets for the visualization of brain pathologies in the human brain $^{7-9)}$. Kollia et al. ${ }^{7)}$ compared 7.0 T MRI with conventional 1.5 T MRI in twelve consecutive patients with multiple sclerosis. The authors reported that 7.0 T MRI was well tolerated and provided better visualization of multiple sclerosis in the gray matter. Lupo et al. ${ }^{8)}$ assessed the feasibility of the susceptibilityweighted imaging technique with 7.0 T MRI. The authors suggested that unique forms of contrast in 7.0 T susceptibilityweighted imaging may be useful for assessing the response to both radiation and anti-angiogenic therapies among patients with brain tumors. Moenninghoff et al. ${ }^{9)}$ reported that 7.0 T MRI offers a more detailed depiction of micro-vascularity and necrosis within astrocytomas because of higher achievable spatial resolution and increased sensitivity for susceptibility contrast compared with 1.5 T MRI. However, there is no previous report regarding 7.0 T MRI in patients with intracranial meningioma.

Although the diagnostic accuracy of conventional MRI is high in meningioma, the information regarding detailed characteristics of meningioma, such as tumor consistency, tumor vasculature and the tumor-brain interface are very important in presurgical planning. In that sense, 7.0 T MRI could provide a more detailed visualization of the tumor-brain interface and intra- or peri-tumoral vasculature compared with conventional MRI.

The presence of peri-tumoral edema correlates well to the loss of a patent arachnoid plane between the meningioma and surrounding normal brain ${ }^{3,6}$. The breakage of the arachnoid plane between the meningioma and brain should be thoroughly evaluated and recognized to properly plan the extent of resection, with minimal injury to the adjacent brain parenchyma, especially in meningiomas that grow against eloquent regions of the brain. In this context, 7.0 T MRI is advantageous when delineating the tumor-brain interface and the neighboring compressed cortex. Our 7.0 T MRI results showed that peri-tumoral edema developed from areas without a cerebrospinal fluid cleft, but this finding could not be perceived in 1.5-T MRI (Fig. 2).

In the resection of meningioma, the veins around tumors must be preserved at all costs ${ }^{5}$. In many cases, cerebral angiography is recommended for the evaluation of feeding vessels, adjacent veins and dural sinuses. The 7.0 T MRI showed detailed information regarding cortical draining veins, small veins around the tumor and intra-tumoral vessels that could not be visualized if the flow is low in $1.5 \mathrm{~T}$ MRI. The 7.0-T MRI is able to display the intra- and peri-tumoral vasculature (Fig. 1) and the cortical venous draining system (Fig. 5) in a T2-weighted image in greater detail in comparison with the current standard 1.5 T MRI. The vessel appearance closely resembles angiography, which is an accepted gold-standard mechanism of intracerebral vessel visualization (Fig. 3).

However, as a result of higher susceptibility in 7.0 T MRI, the image quality of skull base lesions could be reduced compared with 1.5 T MRI, as in case 4 (Fig. 5). Local magnetic field inhomogeneity close to the skull base and the paranasal sinuses and vascular shadows of carotid forks inevitably cause a decline in image quality ${ }^{1)}$. The adjustment of sequence parameters will be required in the future to overcome these spatial artifacts of 7.0 T MR.

\section{CONCLUSION}

7.0 T MRI in patients with meningiomas could provide useful information in surgical strategy, such as the peri-tumoral vasculature and the tumor-brain interface.

\section{- Acknowledgements}

We thank Lauren Seungleal Paek and Emily Knight for their advice in writing this manuscript in English. This study was supported by a grant of the Korea Healthcare Technology R\&D Project, Ministry for Health, Welfare \& Family Affairs, Republic of Korea (A101446-1011-0000400), and by the National Research Foundation (NRF), the Ministry of Education, Science, and Technology of Korea (2012-0006605). 


\section{References}

1. Abduljalil AM, Robitaille PM : Macroscopic susceptibility in ultra high field MRI. J Comput Assist Tomogr 23 : 832-841, 1999

2. Abduljalil AM, Schmalbrock P, Novak V, Chakeres DW : Enhanced gray and white matter contrast of phase susceptibility-weighted images in ultra-high-field magnetic resonance imaging. J Magn Reson Imaging 18 : 284-290, 2003

3. Bitzer M, Wöckel L, Luft AR, Wakhloo AK, Petersen D, Opitz H, et al. : The importance of pial blood supply to the development of peritumoral brain edema in meningiomas. J Neurosurg $87: 368-373,1997$

4. Cha S, Johnson G, Wadghiri YZ, Jin O, Babb J, Zagzag D, et al. : Dynamic, contrast-enhanced perfusion MRI in mouse gliomas : correlation with histopathology. Magn Reson Med 49: 848-855, 2003

5. Giombini S, Solero CL, Lasio G, Morello G : Immediate and late outcome of operations for Parasagittal and falx meningiomas. Report of 342 cases. Surg Neurol 21 : 427-435, 1984

6. Ide M, Jimbo M, Kubo O, Yamamoto M, Imanaga H : Peritumoral brain edema associated with meningioma--histological study of the tumor margin and surrounding brain. Neurol Med Chir (Tokyo) 32: 65-71, 1992

7. Kollia K, Maderwald S, Putzki N, Schlamann M, Theysohn JM, Kraff O, et al. : First clinical study on ultra-high-field MR imaging in patients with multiple sclerosis : comparison of $1.5 \mathrm{~T}$ and 7T. AJNR Am J Neuroradiol $30: 699-702,2009$

8. Lupo JM, Banerjee S, Hammond KE, Kelley DA, Xu D, Chang SM, et al. : GRAPPA-based susceptibility-weighted imaging of normal volunteers and patients with brain tumor at 7 T. Magn Reson Imaging $27: 480$ 488, 2009

9. Moenninghoff C, Maderwald S, Theysohn JM, Kraff O, Ladd ME, El Hindy N, et al. : Imaging of adult astrocytic brain tumours with $7 \mathrm{~T}$ MRI : preliminary results. Eur Radiol 20 : 704-713, 2010

10. Norris DG : High field human imaging. J Magn Reson Imaging 18 : 519-529, 2003

11. Thomas DL, De Vita E, Roberts S, Turner R, Yousry TA, Ordidge RJ : High-resolution fast spin echo imaging of the human brain at $4.7 \mathrm{~T}$ : implementation and sequence characteristics. Magn Reson Med 51 : 1254-1264, 2004 